\title{
Hipotiroidismo congénito, la primera causa de retraso mental prevenible: un desafio para la medicina preventiva
}

\author{
Sergio Andrés Ojeda-Rincón* \\ Édgar Felipe Gualdrón-Rincón** \\ Nicolás Andrés García-Rueda** \\ Diego Felipe Sarmiento-Villamizar** \\ Nathalia Stefania Parada-Botello** \\ Santiago Andrés Gelves-Díaz** \\ Germán Ricardo Rubio-Guerrero**
}

*Estudiante de V año de Medicina de la Universidad Industrial de Santander. Miembro de la Sociedad Estudiantil de Investigación Médica SEIMED-UIS. Escuela de Medicina. Facultad de Salud. Universidad Industrial de Santander. Bucaramanga. Santander. Colombia.

**Estudiante de III año de Medicina de la Universidad Industrial de Santander. Miembro de la Sociedad Estudiantil de Investigación Médica de la Universidad Industrial de Santander SEIMED-UIS. Escuela de Medicina. Facultad de Salud. Universidad Industrial de Santander. Bucaramanga. Santander. Colombia.

Correspondencia: Sergio Andrés Ojeda Rincón. Calle 18 \# 32C- 19. Bucaramanga. Santander. Colombia. Teléfono: 3164513930. Correo electrónico: saor0507@gmail.com.

\section{RESUMEN}

El hipotiroidismo congénito es la enfermedad endocrina más frecuente en neonatos y puede ser de manifiesto clínico o subclínico. Es la principal causa de retraso mental tratable y su pronóstico radica en el tamizaje temprano y la instauración oportuna del tratamiento, por lo cual en Colombia se considera necesaria la implementación de un esquema de tamizaje neonatal con una técnica apropiada y de cobertura nacional, equiparable al de otros países. El objetivo de este artículo es realizar una revisión y análisis de la literatura médica existente sobre el hipotiroidismo congénito centrado en la definición, epidemiología, factores etiológicos, tamizaje, diagnóstico y tratamiento; mediante la búsqueda de artículos en las bases de datos PUBMED, EMBASE, REDALYC, OVID, MEDLINE, DYNAMED y CLINICAL KEY (acceso: mayo 2015). Además se realizó revisión de los informes del Instituto Nacional de Salud en el año 2013 y 2014, de las guías europeas de la sociedad de endocrinología pediátrica 2014, de la guía mexicana de hipotiroidismo congénito neonatal 2008 y del consenso colombiano para el diagnóstico y manejo de enfermedades tiroideas. MÉD UIS. 2016;29(1):53-60.

Palabras clave: Hipotiroidismo Congénito. Tamizaje Neonatal. Epidemiología.

\section{Congenital hypothyroidism, the first cause of preventable mental retardation: a challenge for preventive medicine}

\section{ABSTRACT}

Congenital hypothyroidism is the most common endocrine disease in newborns and can be clinical or subclinical. It is the leading cause of treatable mental retardation and its prognosis is in the early screening and timely initiation of treatment; this is the reason why in Colombia the implementation of a neonatal screening with appropriate national coverage technique, comparable to those in other countries, is considered necessary. The aim of this article is to review and analyse the literature on congenital hypothyroidism focused on the definition, epidemiology, etiological factors, screening, diagnosis and treatment; by search in PubMed, EMBASE, REDALYC, OVID, MEDLINE, and CLINICAL KEY Dynamed data (access: May 2015). Besides reviewing the reports of the National Institute of Health in 2013 and 2014, the European guidelines for pediatric endocrinology society of 2014, Mexico's 2008 guide neonatal congenital hypothyroidism and Colombian consensus for the diagnosis and management of thyroid diseases. MÉD UIS. 2016;29(1):53-60.

Keywords: Congenital hypothyroidism. Neonatal screening. Epidemiology.

¿Cómo citar este artículo?: Ojeda-Rincón SA, Gualdrón-Rincón EF, García-Rueda NA SarmientoVillamizar DF, Parada-Botello NS, Gelves-Díaz SA, et al. Hipotiroidismo congénito, la primera causa de retraso mental prevenible: un desafío para la medicina preventiva. MÉD.UIS. 2016;29(1):53-60.

Artículo recibido el 4 de julio de 2015 y aceptado para publicación el 12 de enero de 2016 


\section{INTRODUCCIÓN}

El término Hipotiroidismo Congénito ( $\mathrm{HC}$ ) fue introducido hace casi 70 años, cuando Radwin y cols describieron en 1949, niños con severas características de discapacidad intelectual y retardo en el crecimiento'. Hoy en día esa definición es obsoleta, puesto que el diagnóstico de HC es realizado incluso antes de la aparición de los síntomas clínicos sobre la medición bioquímica de la Hormona Estimulante de Tiroides (TSH, por sus siglas en inglés) y los niveles de hormona tiroidea ${ }^{2,3}$.

Entre sus formas de presentación, el HC primario, es la forma más común; este el resultado de defectos en el desarrollo de la glándula tiroides, como la agenesia o disgenesia; o debido a las interrupciones de la biosíntesis de la hormona, denominado dihormonogénesis. En el 80\% de los casos, un defecto estructural está presente: una glándula ectópica en ocasiones lingual o en posición craneal; con hipoplasia o en ausencia completa. Por su parte, el HC secundario o central, es causado por deficiencias en la hormona $\mathrm{TSH}$, por ejemplo, en pacientes con anormalidades estructurales o insuficiencia de la glándula pituitaria o el hipotálamo ${ }^{4-6}$. Con el tiempo, los niveles de TSH de corte utilizados en el diagnóstico confirmatorios se han reducido, lo que lleva al diagnóstico de esta enfermedad en los pacientes que presentan elevados niveles de la hormona, sin disminución de las concentraciones de hormona tiroidea periférica $\mathrm{o}$ síntomas clínicos visibles. Esta forma leve de HC se produce como resultado de un aumento transitorio o permanente de los niveles de TSH y ha sido nombrado 'hipotiroidismo subclínico. Sin embargo, dado que esta condición no refleja un verdadero estado de hipotiroidismo, ni causa un defecto en el desarrollo obvio, esta forma sería etiquetada con más precisión como "hipertirotropinemia"4-6.

En Colombia, el HC es la enfermedad endocrina más común en recién nacidos con una frecuencia de uno en 2000 nacidos vivos, sin una diferencia significativa en el género7. El propósito de esta revisión es realizar una búsqueda sistemática en bases de datos centrándose en la definición, epidemiologia, factores etiológicos, tamizaje, manifestaciones clínicas, diagnóstico y tratamiento del HC.

\section{Materiales y Métodos}

La información consignada en la presente revisión fue resultado de la búsqueda en las bases de datos PUBMED, EMBASE, REDALYC, OVID, MEDLINE,
DYNAMED y CLINICAL KEY, utilizando como palabras claves: Neonatal screening y Congenital hypothyroidism. Se incluyeron solo artículos originales y de revisión publicados entre el año 2010 y el 2015. También se revisaron los informes realizados por el Instituto Nacional de Salud de Colombia en el año 2013 y 2014, las guías europeas de la sociedad de endocrinología pediátrica $2014^{11}$, la guía mexicana del hipotiroidismo congénito neonatal $2008^{20}$ y el consenso colombiano para el diagnóstico y manejo de enfermedades tiroideas ${ }^{43}$ obteniendo un total inicial de 139 artículos. Posteriormente se realizó lectura de los mismos y se seleccionaron finalmente 50 artículos. Como criterio de exclusión principal se tuvo en cuenta la falta de relación con el tamizaje y la prevención del HC.

\section{Definición}

El HC es un síndrome clínico y bioquímico resultado de la hipoactividad biológica de las hormonas tiroideas durante la vida fetal, ya sea por un déficit en su producción, resistencia a su acción en los tejidos diana o alteración de su transporte o metabolismo; que compromete principalmente el sistema nervioso central y el sistema esquelético ${ }^{9,10}$. Una de las características para resaltar de este trastorno hormonal es que es la principal causa prevenible de retraso mental ${ }^{11}$. Dentro de sus presentaciones de más difícil detección se encuentra el hipotiroidismo subclínico, el cual se manifiesta con niveles altos de la $\mathrm{TSH}$, niveles normales de las hormonas tiroideas ( $\mathrm{T}_{3}$ y T4) y ausencia de sintomatología ${ }^{12}$.

\section{EPIDEMIOLOGÍA}

El HC es la enfermedad endocrina más común en Recién Nacidos (RN) y la forma de presentación más común del hipotiroidismo en general, el cual es a su vez es el trastorno endocrino más frecuente ${ }^{3}$. La prevalencia de $\mathrm{HC}$ en el mundo es de un afectado por cada 3000 RN vivos y su incidencia es de uno por cada 3000 a 4000 habitantes ${ }^{13-15}$. La incidencia se ve aumentada en embarazos múltiples y en madres mayores de 39 años $^{16}$. Estas cifras pueden ser inexactas debido a la salida hospitalaria temprana, que cambia la edad de realización de la prueba e influye en los resultados del cribado $^{17}$. Generalmente no suelen haber manifestaciones clínicas al nacer, presentándose estas solo en un $5 \%$ de los afectados durante el primer mes, a pesar de que la letalidad de RN con hipotiroidismo alcanzaría el 15 al $20 \% 9,18-20$. Por otro lado, hay una mayor asociación 


\section{ERERO-ABRIL}

entre niños hipotiroideos con síndrome de Down que en niños con función tiroidea normal ( $8 \%$ y 0,12\% respectivamente) ${ }^{21,22}$.

En el $85 \%$ de las veces el $\mathrm{HC}$ es de tipo esporádico mientras que solo un $15 \%$ es hereditario ${ }^{24}$; también el $85 \%$ de casos obedece a algún tipo de disgenesia tiroidea, siendo la ectopia la forma más frecuente y la causa más común de la patología. Otras etiologías ocupan un menor número de casos, como por ejemplo, las mutaciones del gen de la tiroglobulina, un tipo de HC autosómico recesivo con una incidencia estimada de uno por cada $100000 \mathrm{RN}$ $\operatorname{vivos}^{13,20,25}$. Ocurriendo lo contrario en caucásicos, Stoppa y cols (2011) indican que la mayoría de casos en población negra se deben a causas diferentes a la disgenesia tiroidea ${ }^{13}$.

Se ha observado una mayor incidencia de $\mathrm{HC}$ en hispanos y asiáticos, que en nativos americanos y en afrodescendientes, teniendo la población negra, la menor incidencia ${ }^{20}$. Siendo así, en Estados Unidos se ha reportado una incidencia de $\mathrm{HC}$ con tasa de 1 en 3200 y tasa de incidencia de 1 en 1902 nacidos vivos para la población negra, para los blancos de 1 en 1815 y para los asiáticos de 1 en 1016, mientras que la tasa global para este país es de 1 caso por cada 2372 niños ${ }^{9,16}$. Para Canadá la incidencia de HC es de 1 en 3500 nacidos vivos y de acuerdo a la OMS la incidencia en Norteamérica es de 1 en 3600, mientras que en Europa es de entre 1 por cada 6000 a 1 por cada 17000 y en México de 1 en $2800^{20,26}$. Las incidencias en España, Costa Rica y Brasil son 1 en 2875, 1 en 6309 y 1 en 2760 nacidos vivos, respectivamente y para la India abarca valores desde 1 en 2500 a 1 en $2800^{26,27}$.

En lo respectivo a Colombia la enfermedad se presenta aproximadamente en el 50,5\% en hombres, mientras que la incidencia mundial tiene un predominio en la mujer de 2 a 1 , demostrando que en Colombia no hay diferencias en el género en cuanto a la frecuencia de la enfermedad ${ }^{9,20,26}$. En Colombia la frecuencia de la enfermedad se encuentra entre tasas de uno por cada 1886 RN vivos, uno por cada $2500 \mathrm{RN}$ vivos y uno por cada $3348 \mathrm{RN}$ vivos, aunque otros estudios han señalado incidencias de 1 en 2500 , 1 en 2100 y hasta 1 en 3750 niños, esto evidencia que el país aún no cuenta con un valor concreto que indique la ocurrencia del evento, pero sí con un rango aproximado de uno en 2500 a $3500 \mathrm{RN}^{28}$.
Actualmente no existen estudios epidemiológicos de la ciudad de Bucaramanga, sin embargo otros estudios han referido las tasas de la enfermedad en áreas concretas del país como Cali con una incidencia de 1 en 3855 niños nacidos, mientras que en Bogotá, en el hospital San Rafael entre 2010 y 2011, se reportó una prevalencia del $0,9 \%^{29,30}$. En el año 2014, hasta el tercer periodo epidemiológico la enfermedad se presentó a nivel nacional en un $62,1 \%$ en la cabecera municipal, en un $7,6 \%$ en centro poblado y en un $30,3 \%$ en rural disperso; asimismo el departamento con mayor número de casos fue Antioquia con 58, mientras que Santander apareció con 7 casos y aproximadamente 10 departamentos compartieron el último lugar, con un solo caso. Estos diagnósticos se realizaron en un $99,5 \%$ en las primeras cuatro semanas tras el nacimiento y el resto en las semanas quinta a octava 9 .

El hipotiroidismo transitorio se presenta en el $25 \%$ de los RN pre-término, sin embargo la proporción cambia con la región geográfica en relación a la ingesta de yodo; cuando su origen es autoinmune tiene una incidencia de 1 por cada 30,000 nacidos vivos $^{26,31}$.

\section{Etıología}

El HC puede ser permanente o transitorio. (Ver Tabla 1). El HC transitorio, presente generalmente en recién nacidos pre-término, se debe a la inmadurez del sistema tiroideo, presentando bajas concentraciones séricas de T4 libre y total, TSH en rangos normales y respuesta normal de $\mathrm{T} 4$ y TSH a hormona liberadora de tirotropina. Este puede ser inducido por yodo, anticuerpos maternos e incluso, uso de drogas anti-tiroideas maternas ${ }^{32}$. El HC permanente puede ser de etiología primaria, secundaria (central) o periférica. Las causas primarias incluyen disgenesia tiroidea y deficiencias en la producción de hormonas tiroideas las cuales incluyen resistencia a la unión o señalización de $\mathrm{TSH}^{16}$. El HC secundario se debe a la disminución de la síntesis, secreción o bio-actividad de la TSH, que no consigue estimular correctamente una glándula tiroidea completamente normal. ${ }^{6} \mathrm{El} \mathrm{HC}$ periférico resulta de defectos en el transporte, metabolismo de la hormona tiroidea, o resistencia a la acción de la hormona ${ }^{16}$. 
Tabla 1. Clasificación según etiología

\begin{tabular}{|c|c|c|c|}
\hline $\begin{array}{c}\text { Hipotiroidismo } \\
\text { Congénito Primario }\end{array}$ & $\begin{array}{c}\text { Hipotiroidismo } \\
\text { Congénito Secundario o } \\
\text { Central }\end{array}$ & $\begin{array}{c}\text { Hipotiroidismo } \\
\text { Congénito Periférico }\end{array}$ & $\begin{array}{c}\text { Hipotiroidismo } \\
\text { Congénito Transitorio }\end{array}$ \\
\hline $\begin{array}{l}\text { Disgenesia tiroidea: } \\
\text { debido a anomalías } \\
\text { en el desarrollo de } \\
\text { la glándula (ectopia } \\
\text { tiroidea, tiroides } \\
\text { ausente, hipoplasia y } \\
\text { hemigenesia). } \\
\text {-Mutaciones en } \\
\text { los factores de } \\
\text { transcripción: FOXE1, } \\
\text { NKX2.1, PAX-8. }{ }^{16,3,34} \\
\text { Dishormonogénesis } \\
\text { tiroidea: debido a } \\
\text { producción impedida } \\
\text { de hormona tiroidea. } \\
\text { - M u t a i o n e s } \\
\text { a s o c a d a s : } \\
\text { DUOX2, gen de } \\
\text { la tiroperoxidasa } \\
\text { (TPO), Tg (Síntesis } \\
\text { de tiroglobulina), } \\
\text { NIS (simportador } \\
\text { Sodio- Yodo), THOX- } \\
2 \text { (peróxido de } \\
\text { hidrogeno), GNAS1 } \\
\text { (resistencia a TSH). }{ }^{16,3}\end{array}$ & $\begin{array}{l}\text { Deficiencia TSH aislada } \\
\text { - Mutación en el gen de la } \\
\text { subunidad B de TSH). }{ }^{16,6,34} \\
\text { Deficiencia de hormona } \\
\text { liberadora de Tirotropina } \\
\text { (TRH). }{ }^{34} \\
\text { - Síndrome de interrupción } \\
\text { del tallo hipofisario, lesión } \\
\text { hipotalámica. }{ }^{16} \\
\text { Resistencia a TRH. } \\
\text {-Mutación en genes } \\
\text { de receptor de TRH } \\
\text { (TRHR). }{ }^{16,3,6} \text { in debido a } \\
\text { Hipotiroidismo } \\
\text { factores de transcripción } \\
\text { deficientes involucrados } \\
\text { en el desarrollo o función } \\
\text { pituitaria. } \\
\text { - Mutaciones en HESX1, } \\
\text { LHX3, LHX4, PIT1, PROP1, } \\
\text { IGSF1. }{ }^{16,6}\end{array}$ & $\begin{array}{l}\text { Resistencia a hormona } \\
\text { tiroidea. } \\
\text {-Mutación en el } \\
\text { receptor tiroideo B. }{ }^{16,6,3} \\
\text { Anormalidades en } \\
\text { el transporte de la } \\
\text { hormona. } \\
\text { - Síndrome Allan- } \\
\text { He rn d o n-D u d le y } \\
\text { (mutación en el gen } \\
\text { del transportador } \\
\text { de monocarboxilasa } \\
\text { 8[MCT8]). }{ }^{16}\end{array}$ & 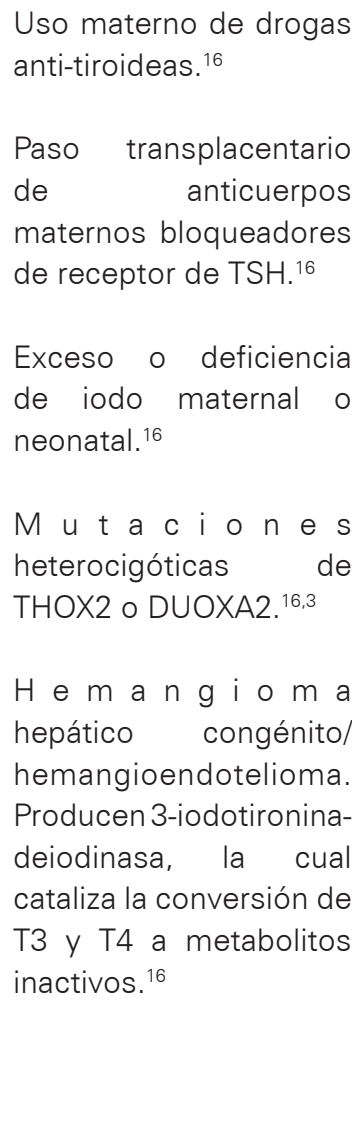 \\
\hline
\end{tabular}

Fuente: ${ }^{16}$ Rastogiy cols, ${ }^{6}$ García y cols, ${ }^{34}$ Adeniran y cols y ${ }^{3}$ Serrano y cols.

\section{TAMIZAJE}

El tamizaje neonatal definido como la identificación pre-sintomática de infantes que poseen alguna condición congénita, se considera el método más efectivo para prevenir el desarrollo severo de secuelas asociadas con hipotiroidismo congénito ${ }^{35,36}$.

Para llevar a cabo el cribado, es necesario la obtención de una muestra de sangre en tarjetas de papel de filtro. Ésta puede ser obtenida de dos formas dependiendo del protocolo establecido en la institución donde se realice, ya sea del cordón umbilical (durante las primeras horas de vida) o del talón (si han transcurrido más de 24 horas después del nacimiento). La recolección de muestras en recién nacidos prematuros es realizada a las 60 horas de vida y en los nacidos a término es realizada en las primeras 24 a 73 horas de vida, siendo esta la mejor ventana ${ }^{38}$. Incluso, algunos programas rutinariamente obtienen una segunda muestra entre la segunda y sexta semanas de edad.

Al final estas muestras son analizadas mediante pruebas de inmunofluoresencia como lo son el fluoruro inmunoensayo inmunométrico ${ }^{39}$ o ELISA. EI examen para el estudio del tamizaje puede realizarse por la combinación de varias concentraciones hormonales, por ejemplo: la combinación de T4 primaria y TSH, o de T4 primaria y TSH secundaria (la segunda muestra de TSH), seguido por la determinación de unión de proteínas de T4. Cabe resaltar que, la forma más sensible de detectar es mediante la prueba de $\mathrm{TSH}^{38}$. A la hora de evaluar e identificar los niveles de hormona en sangre tomada de una muestra de talón, el corte en promedio, en Colombia, se encuentra entre 10-20 mUI/L para TSH. Sin embargo para González y cols (2007), los infantes 
ERERO-ABRIL

que se encuentran con valores superiores a $10 \mathrm{mU} / \mathrm{L}$, deben ser derivados al centro especializado para realizar estudios de confirmación ${ }^{40}$. Para las muestras de cordón umbilical con valores de TSH mayores 15 $\mathrm{mU} / \mathrm{L}$ se realiza una segunda prueba confirmatoria?.

En Colombia, este procedimiento está contemplado en la Resolución 0412 del Ministerio de Salud, utilizando la muestra de cordón umbilical posparto y un formato con los datos de identificación correspondientes 9 . A diferencia de muchos países, la toma universalmente aceptada de sangre de talón para pruebas de tamizaje neonatal no se justifica en Colombia por no tener programas de tamizajes diferentes de hipotiroidismo congénito ${ }^{37}$. El éxito del tamizaje radica en la toma adecuada de muestras y por consiguiente la adecuada discriminación de los neonatos que padecen HC. Por lo que se considera pertinente el conocimiento de la forma en la que se debe realizar (Ver Tabla 2).

Tabla 2. Procedimiento instaurado para la toma de muestra del cordón según el informe epidemiológico del instituto nacional de salud. ${ }^{9}$

\section{Pasos para tomar la muestra del cordón}

1. Posterior a la limpieza correspondiente con solución salina y con un tiempo límite de 20 minutos, se extraen de 0,5 a $1 \mathrm{~mL}$ de sangre de la vena en la parte media del cordón.

2. Con la muestra en la jeringa, se procede a depositar una gota de sangre en cada círculo del papel de filtro a una distancia aproximada de $3 \mathrm{~mm}$. Las gotas deben alcanzar a impregnar la parte posterior del papel.

3. Las muestras se dejan secar a temperatura ambiente durante tres horas. Deben existir como mínimo tres círculos con calidad óptima.

4. Después se deben almacenar en un lugar seco dentro de un sobre de papel.

5. En sitios de temperatura muy alta $\left(14-22^{\circ} \mathrm{C}\right)$, refrigerar de $2-8^{\circ} \mathrm{C}$.

Nota: Se debe disponer de un soporte para que no se apilen las tarjetas de diferentes individuos ni entren en contacto con otras superficies, con el fin de evitar contaminación y alteraciones en el resultado.

Fuente: Misnaza SP, Garzón FE. Informe del evento hipotiroidismo congénito hasta el periodo epidemiológico tres del año 2014. Instituto nacional de Salud, 2012, versión 01, pág. 1-14.

Si la muestra tomada es inadecuada se deberá llevar a cabo una nueva del talón antes del egreso del neonato o citarlo entre el tercer y séptimo día de nacido. Los procedimientos de secado y mantenimiento de la toma son conservados en las condiciones nombradas. Ulterior al análisis correspondiente de la prueba, se clasifica: cuantitativamente el resultado obtenido como caso probable, probable re-tamizado, negativo o confirmado según los valores encontrados; y cualitativamente como positivo verdadero, positivo falso, negativo verdadero y negativo falso ${ }^{37}$ (Ver Tabla 3). Cabe resaltar que aunque la prueba tiene una alta sensibilidad para identificar los casos de HC, Zeinalzadeh y Talebi (2012) recomiendan la toma de una segunda prueba para neonatos en las siguientes condiciones: neonatos prematuros, de bajo peso, prematuros enfermos admitidos a cuidados intensivos, partos múltiples y gemelos del mismo sexo ${ }^{41}$.

Tabla 3. Definición de caso según el informe epidemiológico del instituto nacional de salud de colombia $2014 .^{9}$

\begin{tabular}{|l|l|}
\hline \multicolumn{1}{|c|}{ Definición } & \multicolumn{1}{c|}{ Criterio } \\
\hline Caso negativo & TSH normal, es decir $<20 \mathrm{mUI} / \mathrm{L}$ de sangre de cordón \\
\hline Caso probable & TSH $>20 \mathrm{mUI} / \mathrm{L}$ de sangre de cordón o $15 \mathrm{mUI} / \mathrm{L}$ de sangre del talón \\
\hline Caso probable re-tamizado & Caso probable que se estudia con T4 \\
\hline Caso confirmado & T4 $<42.9 \mu \mathrm{g} / \mathrm{L}$ en suero \\
\hline Caso positivo verdadero & Caso que al re-tamizar se confirma \\
\hline Caso positivo falso & Caso probable que al re-tamizar es normal y se descarta \\
\hline Caso negativo verdadero & Caso negativo que no desarrolla la enfermedad \\
\hline Caso negativo falso & Caso negativo que desarrolla la enfermedad \\
\hline
\end{tabular}

Fuente: Misnaza SP, Garzón FE. Informe del evento hipotiroidismo congénito hasta el periodo epidemiológico tres del año 2014. Instituto nacional de Salud, 2012, versión 01, pág. 1-14. 


\section{Manifestaciones ClíNICAS}

Los pacientes con hipotiroidismo son pacientes que cursan confaciesmuymarcadas, toscas einexpresivas, con párpados y labios gruesos, nariz corta con base deprimida y con los orificios dirigidos hacia delante. Es común también en estos pacientes apreciar una lengua prominente, la cual sobresale de los labios por acumulación de ácido hialuronico9. Al ser una patología que afecta múltiples sistemas, es posible que estos pacientes cursen a nivel cardiovascular con bradicardias sinusales persistentes o presenten manifestaciones gastrointestinales como ictericia, constipación intestinal y tendencia al aumento de peso $^{10}$. A su vez, es frecuente determinar en ellos la presencia de apneas, fatiga acompañada de calambres musculares, hiporreflexia, además de malformaciones en el desarrollo óseo, como cifosis dorsales y ampliamiento de las fontanelas anterior y posterior ${ }^{16}$. La conducta y el desarrollo del paciente hipotiroideo también se ve afectado, son pacientes somnolientos con letargia y retraso en el desarrollo neurológico ${ }^{20}$. Adicionalmente presentan cianosis, hernia umbilical, irregularidades menstruales, piel seca e intolerancia al frío ${ }^{26}$.

\section{Diagnóstico Diferencial}

El diagnóstico diferencial del hipotiroidismo se establece con patologías que asemejan mixedema como las mucopolisacaridosis I y II, junto con la gangliosidosis en la que el signo diferencial es la hepatomegalia o atresia biliar. Asimismo, hay síndromes genéticos como el de Beckwith Wiedeman, Albright y Síndrome de Down, que cursan con facies similares al mixedema facial, puente nasal plano, macroglosia e hipotonía ${ }^{20}$.

\section{TRATAMIENTO}

El manejo del $\mathrm{HC}$ es fundamentalmente farmacológico pues el costo-efectividad es suficiente para sopesar las desventajas y los riesgos de la progresión, sin embargo cabe destacar la importancia de un tratamiento integral y continuo donde se pueda garantizar la adherencia, el seguimiento y el control del comportamiento bioquímico hormonal como a su vez, de la evolución sintomática. Dentro de las opciones farmacológicas es de gran utilidad el manejo con levotiroxina sódica en las presentaciones disponibles para la administración oral $^{23}$. La dosis utilizada varía de un estudio a otro, sin embargo la dosis inicial más recomendada está en un rango entre 10 a $15 \mu / \mathrm{kg} /$ día. ${ }^{8,43}$ La variabilidad de ésta de acuerdo a la edad debe ser tenida en cuenta (Ver Tabla 4).

Tabla 4. Dosificación de levotiroxina recomendada según la edad.

\begin{tabular}{|l|c|}
\hline \multicolumn{1}{|c|}{ EDAD } & DOSIS \\
\hline Hasta el primer mes: & $10-15 \mu / \mathrm{kg} /$ día \\
\hline Segundo mes & $7-10 \mu / \mathrm{kg} /$ día \\
\hline Del tercer al quinto mes & $4-7 \mu / \mathrm{kg} /$ día \\
\hline Del sexto mes al primer año & $4-6 \mu / \mathrm{kg} /$ día \\
\hline Primer y segundo año & $4-6 \mu / \mathrm{kg} /$ día \\
\hline De los 3 a 7 años & $3-4 \mu / \mathrm{kg} /$ día \\
\hline De los 10 a 12 años & $2-3 \mu / \mathrm{kg} /$ día \\
\hline Mayores de 12 años & $2 \mu / \mathrm{kg} /$ día \\
\hline
\end{tabular}

Fuente: Fullana A, Barona-Vilar C, E Mayayo Dehesa. ${ }^{8,10}$

Adicionalmente es importante mencionar que la hormona estimulante de tiroides logra estabilizarse alrededor de la sexta a octava semana una vez iniciado el tratamiento, por lo que se debe hacer un seguimiento midiendo los valores séricos de esta cada seis a ocho semanas para decidir si se debe reajustar la dosis, y además hacer una valoración clínica cuidadosa ${ }^{43}$. Dicha valoración incluye una consulta con endocrinólogo pediatra en la cual se incluyan una historia clínica y un examen físico completo, evaluando también las medidas en suero de las concentraciones hormonales de T4 y los títulos de anticuerpos antitiroideos si los hay. A su vez, se sugiere un estudio de la edad ósea (para conocer el efecto durante la vida fetal), y una exploración de tiroides con yodo radiactivo para establecer si hay permanencia de la enfermedad ${ }^{10}$. Gonzáles y cols recomiendan realizar el seguimiento hormonal, después de iniciado el tratamiento a las dos y cuatro semanas, luego cada dos meses durante los primeros seis meses de vida, posteriormente cada cuatro meses entre los 6 y 36 meses de vida, y después una monitorización anual ${ }^{50}$. Según la guía mexicana de manejo de hipotiroidismo congénito el monitoreo debe realizarse durante los tres primeros años de tratamiento y en lo posible deben mantenerse niveles de TSH entre 0,5 y 2 mUL, y reevaluar si es necesario continuar o modificar la conducta terapéutica con levotiroxina ${ }^{20}$. En casos especiales como en el embarazo el suministro de levotiroxina puede llegar a ser un factor protector para no desarrollar hipotiroidismo congénito en el bebé, debido a que normalmente la necesidad de hormona tiroidea aumenta y es necesario dar suplemento ${ }^{42,44}$. 


\section{Pronóstico de la Enfermedad}

El curso y la evolución de la enfermedad está destinado a traer graves consecuencias en el desarrollo neuro-cognitivo principalmente y en el desarrollo de múltiples sistemas por lo que la carga de discapacidad mental y general es realmente considerable, sin embargo el manejo temprano y oportuno, y el seguimiento estricto permiten mejorar, prevenir y hasta evitar las consecuencias negativas de la enfermedad. El manejo farmacológico y clínico permite mejorar el IQ rápidamente en comparación con los niños que no han recibido tratamiento, incluso aquellos que han sido tratados podrían alcanzar un crecimiento, desarrollo y peso normal para su edad ${ }^{45}$. Además, se ha informado mejora en el estado de ánimo y la función neuropsicológica en pacientes que han sido tratados con una combinación de hormonal T4 y T349; y mejora en los cambios del desarrollo psicomotor y del lenguaje con el empleo oportuno y temprano de levotiroxina ${ }^{46,48}$. Dentro de las consecuencias negativas del tratamiento por ejemplo podemos encontrar variaciones en el peso que aún no se han correlacionado con niveles hormonales de THS; y compromiso de la inteligencia, donde se evidencian disminuciones del IQ, de 5 a 10 puntos ${ }^{47}$.

\section{CONCLUSIÓn}

El hipotiroidismo es la más frecuente de todas las enfermedades endocrinas y su forma congénita es la presentación más común. La etiología del HC en la mayoría de los casos es la ectopia tiroidea; y su incidencia aproximada en Colombia es uno en 2500 a 3500 RN. Además es la principal causa de retraso mental tratable, y su pronóstico depende de un tamizaje precoz y la instauración del tratamiento. El tamizaje neonatal es la principal herramienta para detectar el HC; para que esta técnica sea efectiva y equiparable a las realizadas en otros países con mejor vigilancia de la enfermedad, hay que tener en cuenta el lugar de extracción de muestra, sea el talón o el cordón umbilical; que el procedimiento se ejecute correctamente; y que se haga el procesamiento adecuado de la muestra, con el fin de garantizar los altos estándares de calidad y un resultado certero que contribuya al buen juicio del clínico. El tratamiento debe ser oportuno y continuo, asegurando una adherencia y conciencia de la enfermedad tanto en pacientes como en sus familiares. En Colombia la captura diagnóstica de esta enfermedad no ha alcanzado la cobertura óptima del tamizaje en el total de la población, esto evidencia la ausencia de datos epidemiológicos que describan la frecuencia y la distribución de la enfermedad en la región. Por tanto, es importante la realización de nuevos estudios para analizar la situación actual del HC en el país que a su vez permitan esclarecer si lo individuos afectados alcanzan el crecimiento y el desarrollo neurointelectual comparable con el que potencialmente lograrían en ausencia de HC. Además es imprescindible reevaluar la efectividad de los sistemas de tamizaje y diagnóstico dentro del sistema de salud de Colombia.

\section{REFERENCIAS}

1. Radwin, L. S., Michelson, J. P., Berman, A. B. \& Kramer, B. End results in treatment of congenital hypothyroidism; follow-up study of physical, mental and behavioral development. Am J Dis Child. 1949;78(6):821-43.

2. Fisher, D. A. et al. Screening for congenital hypothyroidism: results of screening one million North American infants. J Pediatr. 1979;94(5):700-5.

3. Illig, R. \& Gitzelmann, R. Screening for congenital hypothyroidism. J Pediatr. 1977;91(2):348-9.

4. Clerc, J. et al. Scintigraphic imaging of paediatric thyroid dysfunction. Horm Res. 2008;70(1):1-13.

5. Bubuteishvili, L., Garel, C., Czernichow, P.\& Léger, J. Thyroid abnormalities by ultrasonography in neonates with congenital hypothyroidism. J Pediatr. 2003; 143(6):759-64.

6. Marinovic, D., Garel, C., Czernichow, P. \& Léger, J. Ultrasonographic assessment of the ectopic thyroid tissue in children with congenital hypothyroidism. Pediatr Radiol. 2004;34(2):109-13.

7. Bermúdez AJ, Hernández LS, González NE. Tamizaje neonatal de hipotiroidismo congénito, vigilancia por el laboratorio. Ministerio de la protección social, Instituto Nacional de Salud, 2004.

8. Fullana A, Barona-Vilar C. Cribado neonatal del hipotiroidismo congénito. Una gran aportación a la salud pública de las últimas décadas. Med Clin (Barc). 2010; 134(7):307-9.

9. Misnaza SP, Garzón FE. Informe del evento hipotiroidismo congénito hasta el periodo epidemiológico tres del año 2014. Instituto Nacional de Salud, 2012, Versión 01, pág. 1-14.

10. E Mayayo Dehesa. Hipotiroidismo y bocio. Protoc Diagn Ter Pediatr. 2011;1:150-65.

11. Léger J, Olivieri A, Donaldson M, Torresani T, Krude H, van Vliet $\mathrm{H}$, et al. European Society for Paediatric Endocrinology Consensus Guidelines on Screening, Diagnosis, and Management of Congenital Hypothyroidism. J Clin Endocrinol Metab. 2014; 99(2): 363-84.

12. Chen X, Yu-Feng Q, Xue-Lian Z, Ru-Lai Y, Yu-Hua S, Hua-Qing $\mathrm{M}$, et al. Diagnosis and treatment of subclinical hypothyroidism detected by neonatal screening. World J Pediatr. 2011; 7(4):350-4.

13. Stoppa S, Van G, Deladoëy J. Variation by ethnicity in the prevalence of congenital Hypothyroidism due to thyroid dysgenesis. Thyroid. 2011; 21(1):13-8.

14. Toublanc J. Comparision of epidemiological data on congenital hypothyroidism in Europe with those of other parts in the world. Horm Res. 1992; 38(5-6):230-5.

15. Felice M, Lauro R. Thyroid Development and Its Disorders: Genetics and Molecular Mechanisms. Endocr Rev. 2004; 25(5):722-46.

16. Rastogi MV, LaFranchi SH. Congenital hypothyroidism. Orphanet J Rare Dis. 2010;5(17):1-22.

17. Marrero-González N, González E, Frómeta A, Baloy A, Castells E, Pérez P, et al. Influencia de la edad en los resultados del cribado neonatal. Rev Mex Patol Clin. 2004;51(4):220-5.

18. Martinez M, Orozco M. Hipotiroidismo Congénito. Reporte de un Caso Diagnosticado por Tamiz Neonatal. Bol Clin Hosp Infant Edo Son. 2008;25(2):115-8. 
19. Thvillum M, Brandt F, Hegedüs T. A review of the evidence for and against increased mortality in hypothyroidism. Nat. Rev. Endocrinol. 2012;8:417-24.

20. Centro Nacional de Excelencia Tecnológica en Salud. Prevención, diagnóstico y tratamiento del hipotiroidismo congénito neonatal en el primer nivel de atención. México: CENETEC;2008.

21. Razavi Z, Yavarikia A, Torabian S. Congenital Anomalies in Infant with Congenital Hypothyroidism. Oman Med J. 2012;27(5):364-7.

22. Purdy IB, Singh N, Brown WL, Vangala S, Devaskar UP. Revisiting early hypothyroidism screening in infants with Down syndrome. J Perinatol. 2014;34(12):936-40

23. Rose S, Brown R, Foley T, Kaplowitz P, Sundararajan S, Varma S, et al. Update of newborn Screening and Teraphy for Congenital Hypothyroidism. Pediatrics. 2006;117(6):2290-303

24. Rodriguez G, Assad J, García M, Arias M, Zapata M. Factores que influyen en el diagnóstico e inicio de tratamiento, oportuno en el hipotiroidismo congénito. Pediatr Mex. 1999;1.2(7):19-24.

25. Liu S, Zhang S, Li W, Zhang A, Qi F, Zheng G, et al. Clinical and Genetic Analysis of a Compound Heterozygous Mutation in the Thyroglobulin Gene in a Chinese Twin Family With Congenital Goiter and Hypothyroidism. Twin Res Hum Genet. 2012;15(1):126-32.

26. Bermúdez AJ, Hernández LS, González NE. Tamizaje neonatal de hipotiroidismo congénito, vigilancia por el laboratorio. Colombia: Instituto Nacional de Salud; 2004.

27. Nair P, Sobhakumar S, Kailas L. Diagnostic Re-evaluation of Children with Congenital Hypothyroidism. Indian Pediatr. 2010;47(9):757-60

28. Bermúdez A, Gonzales N, Rosero M, Escobar J. Protocolo de vigilancia del hipotiroidismo congénito.Colombia: Instituto Nacional de Salud;2008

29. Bernal M, Caldas M, Bonilla R, Chamorro G, Matallana A. Tamización para hipotiroidismo congénito en Cali y constitución de un centro piloto de referencia para la identificación temprana de la enfermedad. Colomb Med. 2003;34(1):40-6

30. Vanegas N. Prevalencia de Hipotiroidismo congénito diagnosticado con tamizaje en recién nacidos del Hospital San Rafael de Bogotá entre el año 2010 al 2011. [Tesis de posgrado]. Bogotá D.C.: Universidad Militar Nueva Granada; 2012.

31. Gobernación del Tolima, secretaria de salud departamental dirección de salud pública. Tamizaje neonatal para hipotiroidismo congénito

32. Martínez SN, Morales P, Hernández MG. Caso clínico: Hipotiroidismo congénito, diagnóstico oportuno. Rev Invest Med Sur Mex. 2012; 19(3): 184-6.

33. Grob LF, Martínez-Aguayo A. Hipotiroidismo congénito: un diagnóstico que no debemos olvidar. Rev Chil Pediatr. 2012; 83(5): 482-91.

34. Adeniran, K. A, Limbe M. Review Article on Congenital Hypothyroidism and Newborn Screening Program in Africa; the
Present Situation and the Way Forward. Thyroid Disorders Ther 2012; 1(102):1-4.

35. Pitt, J. Newborn Screening. Clin Biochem Rev. 2010; 31(2):57-68.

36. Sanghvi U, Diwakar KK. Universal Newborn Screening for Congenital Hypothyroidism. Indian J Pediatr. 2008; 45(4)331-2.

37. Misnaza SP. Informe final hipotiroidismo congénito del año 2013. Instituto Nacional de Salud vigilancia y control en salud pública. 2012; versión 01:1-16.

38. Grüters A, Krude H. Detection and treatment of congenital hypothyroidism. Nat Rev Endocrinol. 2012; 8(2):104-13.

39. Goel P, Kahkasha, Narang S, Gupta BK, Goel K. Evaluation of Serum Prolactin Level in Patients of Subclinical and Overt hypothyroidism. J Clin Diagn Res. 2015 ;9(1):BC15-7

40. González V, Santucci Z, Pattin J, Apezteguía M, Borrajo G. Programa de pesquisa neonatal de hipotiroidismo congénito de la provincia de Buenos Aires: 1.377.455 niños evaluados en diez años de experiencia. Arch Argent Pediatr. 2007; 105(5):390-7.

41. Zeinalzadeh A, Talebi M. Neonatal screening for congenital hypothyroidism in East Azerbaijan, Iran: the first report. J Med Screen. 2012;19(3):123-6

42. Osborn, DA. Hunt, RW. Prophylactic postnatal thyroid hormones for prevention of morbidity and mortality in preterm infants. Cochrane Database Syst Rev. 2007; 24(1):CD005948.

43. Asociación Colombiana de Endocrinología Santafé de Bogotá Colombia. Consenso colombiano para el diagnóstico y manejo de enfermedades tiroideas. Acta Med Colomb. 1999;(24):4 159-74.

44. Alexander E, Marqusee E, Jennifer L ,Jarolim P. Fsicher G, Reed Larsen P. Timing and Magnitude of Increases in Levothyroxine Requirements during Pregnancy in Women with Hypothyroidism. N Engl J Me. 2004; (351):241-9.

45. The National Health Service (NHS) UK Newbor Screening Programme Centre's Health Professional Handbook.2012;(1):5-46

46. Grosse SD, Van Vliet G. Prevention of intellectual disability through screening for congenital hypothyroidism: how much and at what level?. Arch Dis Child. 2011;96(4):374-9.

47. Cameo T, Gumer LB, Williams KM, Gomez J, McMahon DJ, Oberfield SE. A retrospective review of newborn screening for congenital hypothyroidism and newborn thyroid disease at a major medical center. Clin Pediatr (Phila). 2013;52(11):1054-8.

48. Marti S, Alvarez M, Simoneau-Roy J, Leroux S, Van Vliet G, Robaey P. Effects of early high-dose levothyroxine treatment on auditory brain event-related potentials at school entry in children with congenital hypothyroidism. Horm Res. 2006;66(5):240-8.

49. Cassio A, Cacciari E, Cicognani A, Damiani G, Missiroli G, Corbelli E, et al. Treatment for Congenital Hypothyroidism: Thyroxine Alone or Thyroxine Plus Triiodothyronine?. Pediatrics. 2003;111(5 Pt 1):1005-1060.

50. Marrero N, Rodríguez C. Hipotiroidismo Congénito: historia e impacto del tamizaje. Rev Biomed. 2000;11(4):283-92. 